\title{
Laser curing of screen and inkjet printed conductors on flexible substrates
}

\author{
Yang Wei, Yi Li, Russel Torah and John Tudor \\ University of Southampton, \\ Southampton, United Kingdom, \\ SO17 3BJ \\ Tel: $+44(0) 2380592126$ \\ Fax: +44(0)2380592901 \\ y.wei@soton.ac.uk
}

\begin{abstract}
Copper nanoparticle (NP) inks offer lower cost conductors compared with the more common silver conductors used for flexible electronics applications. However, oxidation occurs during conventional oven-curing limiting their use. This paper reports screen/inkjet printed copper conductors, cured using a laser, on flexible Kapton (Polyimide), PET (Polyethylene terephthalate), paper and polyester/cotton fabric in ambient air. The results show that copper NP inks can be laser sintered without oxidation or damage to these flexible substrates.
\end{abstract}

Keywords-Laser curing, screen-printing, inkjet printing, conductors, flexible substrates, test structure

\section{INTRODUCTION}

Silver ink is frequently used in flexible electronics due to its high conductivity $\left(6.30 \times 10^{7} \mathrm{~S} / \mathrm{m}\right)$. Even if oxidised, silver oxide $\left(\mathrm{Ag}_{2} \mathrm{O}\right)$ is conductive and the oxidation does not significantly affect the conductivity. Copper is an alternative conductor which has a bulk conductivity of $5.96 \times 10^{7} \mathrm{~S} / \mathrm{m}$. However, copper is typically 10 times cheaper than silver [1] and therefore offers significant cost savings as a replacement. However, copper oxidises at room temperature resulting in copper oxide $(\mathrm{CuO})$ which is an insulator that significantly reduces the conductivity to the $\mu \mathrm{S} / \mathrm{m}$ range.

There are several solutions to reduce the copper oxidation. Previously the copper was sintered in a nitrogen atmosphere and at temperatures above $250{ }^{\circ} \mathrm{C}$. However, this process is not ideal for flexible or fabric based circuits or roll to roll processing. DuPont uses silver-coated copper nanoparticles [2] and therefore the ink can be cured using the same method as a traditional silver ink. Novacentrix use an ink based on copper oxide mixed with reducing agents [3]. They subsequently use photonic curing to sinter the copper particles in air and convert the copper oxide to copper via the reducing agent. This copper ink also shows good compatibility with flexible substrates (e.g. PET). Intrinsiq Ltd. uses an organic shell on the copper NPs to prevent oxidation; this shell is removed when laser cured [4].

Substrates, such as Kapton and PET, are commonly used for printed electronics, offering flexibility and compatibility with printed materials. There are several deposition methods for copper ink: inkjet printing [5], soft lithography [6] and screen-printing [7]. Screen-printing is able to deposit inks with a broad rheology (1-300 Pa.s) [8], whereas inkjet printing has a very narrow and low viscosity range (0.01 to $0.04 \mathrm{~Pa} . \mathrm{s})$ [9].

The deposited copper ink can be either thermally in an oven or optically cured. Thermally curing has fewer restrictions on ink formulation; the thermal energy removes the solvent and, if sufficiently high, will sinter the copper NPs. This typically requires sintering above $200{ }^{\circ} \mathrm{C}$, potentially harming some flexible substrates. Laser curing only heats a specific area therefore avoiding excessive thermal energy transmission to the substrate thus reducing damage. Laser curing uses the intense thermal energy generated from a laser diode source to sinter the NPs to form a continuous conductor. The time and energy required to cure a printed copper layer depends on its dimensions and the substrate. The thermal conductivity of the substrate affects the achievable conductivity of the copper. The thermal conductivity of the substrate should ideally be less than that of the printed ink so energy is more efficiently transferred to the ink to evaporate the solvent and sinter the NPs [10].

This paper reports the results of laser curing screen/inkjet printed copper tracks onto four flexible substrates: Kapton, PET, paper and polyester/cotton fabric. Printed tracks are cured with different curing speeds at different laser powers. The conductivity of the cured copper is evaluated as a function of the laser power and the curing speed.

\section{EXPERIMENTAL PROCEDURES}

\section{A. Laser setup}

A LAPS60 laser system from Intrinsiq Materials Ltd was used in this research, with a $60 \mathrm{~W}$ maximum power at $808 \mathrm{~nm}$ wavelength with a rectangular beam. The laser power can be adjusted by changing the drive current to a maximum of $60 \mathrm{~A}$. The system is mounted on a bespoke, PC controlled, XYZ stage where the laser remains fixed in $X Y$ and the substrate moves beneath it. The focus of the laser is experimentally adjusted using the $\mathrm{Z}$ stage.

\section{B. Substrate and material selection}

Kapton, PET, paper and polyester/cotton fabric were chosen as the substrates. Kapton, PET and paper are often used as the substrates for printable/flexible electronics and fabric is a 
TABLE I. THERMAL CONDUCTIVITY OF SELECTED SUBSTRATE MATERIALS.

\begin{tabular}{|c|c|c|c|c|}
\hline Substrate & Kapton & PET & Paper & Fabric \\
\hline $\begin{array}{c}\text { Thermal } \\
\text { conductivity } \\
\text { (W/mK) }\end{array}$ & 0.20 & 0.15 & 0.01 & $0.18^{*}$ \\
\hline $\begin{array}{l}\text { *No thermal conductivity information on selected fabric is available; the value provided is based } \\
\text { on the percentage of each in the fabric. }\end{array}$
\end{tabular}

suitable substrate for wearable electronics. These substrates were cut to $100 \times 100 \mathrm{~mm}$. Kapton and PET were cleaned using IPA prior to printing and the paper and fabric were used as is. Table 1 shows the thermal conductivity of each substrate.

The thermal conductivities of the substrates are much lower than copper $(385 \mathrm{~W} / \mathrm{mK})$ and therefore the printed copper inks are more likely to be successfully laser cured. Both screen (CP003/004) and inkjet copper (CP-002) inks are provided by Intrinsiq Materials. Both inks consist of copper micro/nano particles with organic shells blended with appropriate solvents.

\section{Pattern and printing}

To evaluate the conductivity of the printed copper, simple printed strip patterns are used. A DEK 248 printer was used to screen-print strip lengths of $80 \mathrm{~mm}$ with widths from 1 to 6 mm. A Dimatix DMP-2831 was used to inkjet print strips of length $80 \mathrm{~mm}$ and width $2 \mathrm{~mm}$. All prints were in ambient air and the printed tracks were first dried in a thermal oven to remove the solvent.

\section{RESULTS}

Printed copper tracks are cured with the laser current being adjusted from 30 to $60 \mathrm{~A}$ at $10 \mathrm{~A}$ intervals. The traverse speed of the stage was initially set to $50 \mathrm{~mm} / \mathrm{s}$ and then either increased or decreased depending on results. The laser line scans the printed sample once along the longest edge with a 25 $\mathrm{mm}$ focal length.

\section{A. Screen printed copper tracks}

The printed copper tracks were first dried in a conventional oven at $60{ }^{\circ} \mathrm{C}$ for 30 minutes to remove the solvent before laser curing. When curing at $60 \mathrm{~A}$ on Kapton and PET substrates, the copper tracks have poor adhesion resulting in the layers

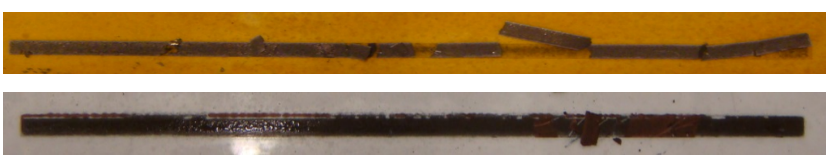

Figure 1: Example of flaked copper tracks on Kapton (top) curing PET (bottom) while cured at $60 \mathrm{~A}$.

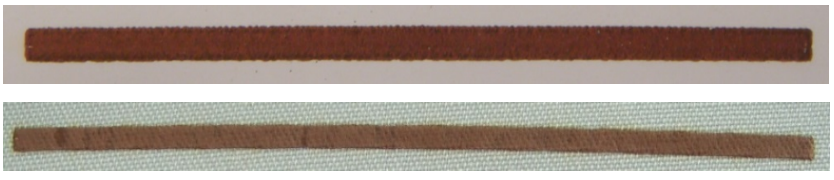

Figure 2: Example of cured CP-003 copper tracks on paper (top) and fabric (bottom) at $60 \mathrm{~A}$. flaking off. However, on paper and fabric they do not flake off because both paper and fabric are more absorbent than Kapton and PET, thus improving the adhesion between the layer and

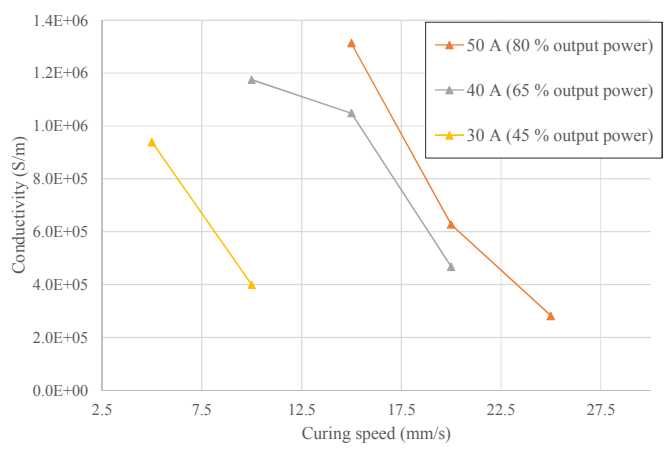

(a)

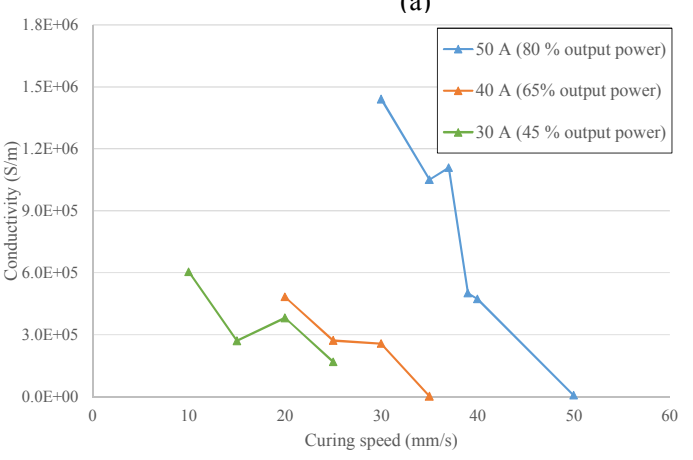

(b)

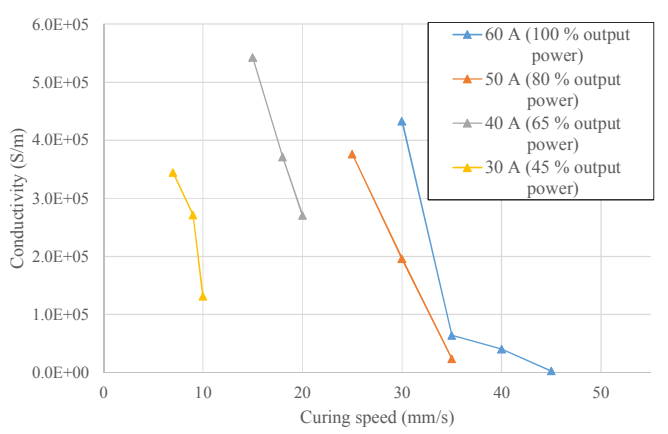

(c)

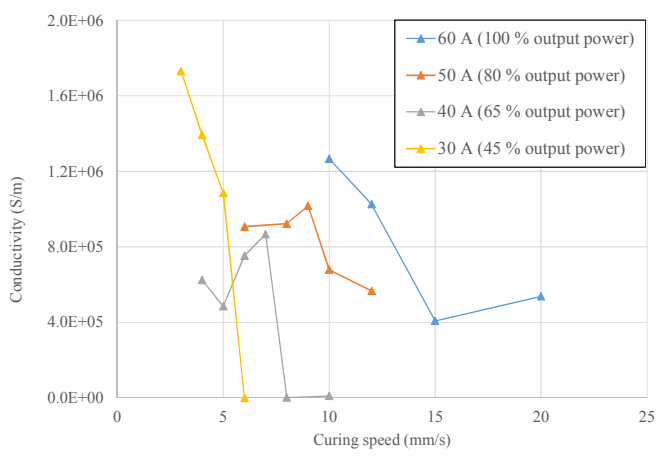

(d)

Figure 3: Conductivity of screen-printed CP-003 copper vs curing speed on (a) Kapton; (b) PET; (c) Paper and (d) polyester/cotton fabric. 
the substrate. Figure 1 and Figure 2 show examples of the copper tracks on the four substrates.

Conductivities are calculated based on the measured resistance and the dimensions of individual tracks on each substrate and are shown in Figure 3.

Kapton - No conductivity can be obtained from the tracks cured at $60 \mathrm{~A}$. The best conductivity of the copper track on Kapton is $1.3 \times 10^{6} \mathrm{~S} / \mathrm{m}$ obtained at $50 \mathrm{~A}$ with a $15 \mathrm{~mm} / \mathrm{s}$ curing speed.

PET - No conductivity can be obtained while curing at 60 A because the cured layers flaked off. The best conductivity of the copper track on PET is $1.4 \times 10^{6} \mathrm{~S} / \mathrm{m}$ obtained at $50 \mathrm{~A}$ with a $30 \mathrm{~mm} / \mathrm{s}$ curing speed.

Paper - The best conductivity on paper is $5.4 \times 10^{5} \mathrm{~S} / \mathrm{m}$ obtained at 40 A with a $15 \mathrm{~mm} / \mathrm{s}$ curing speed.

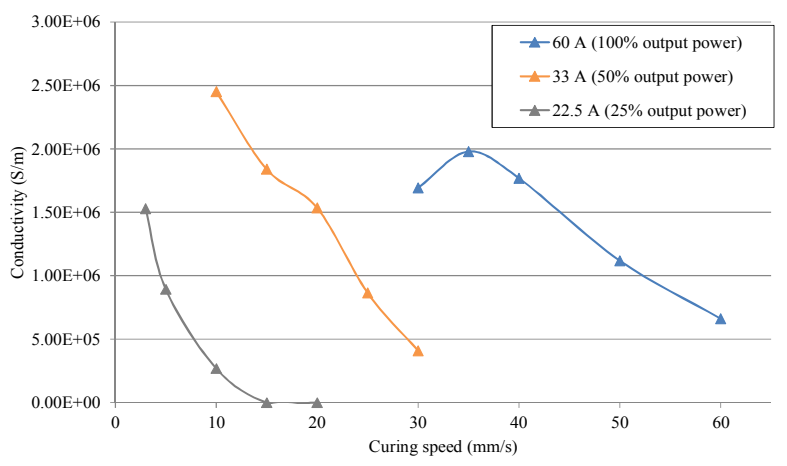

(a)

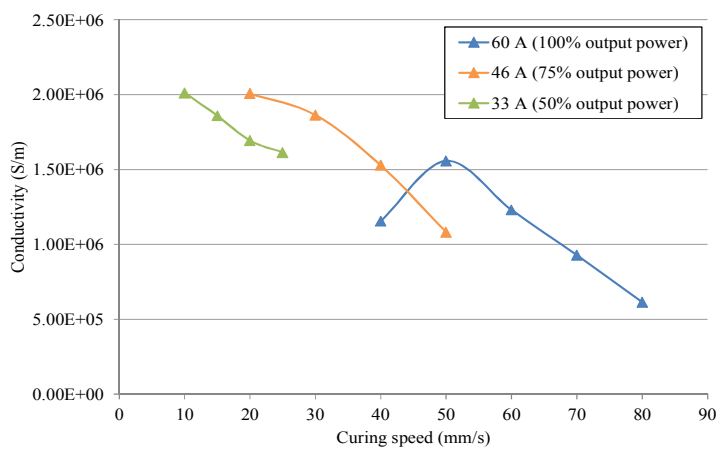

(b)

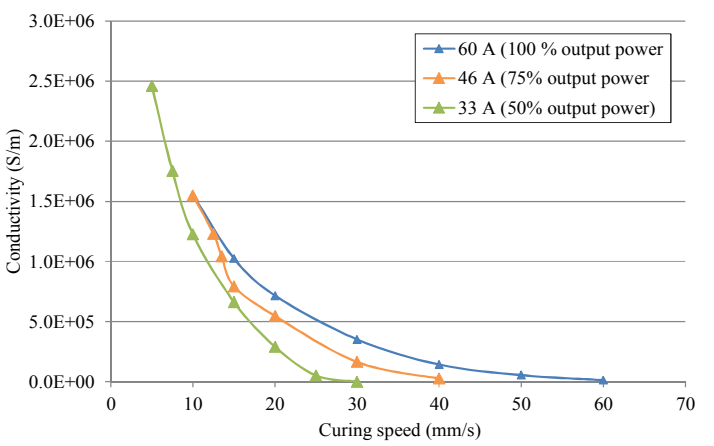

(c)

Figure 4: Conductivity of screen-printed CP-004 copper vs curing speed on (a) Kapton; (b) Paper and (c) polyester/cotton fabric.

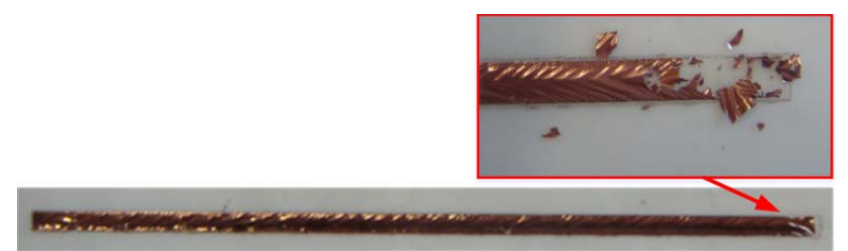

(a)

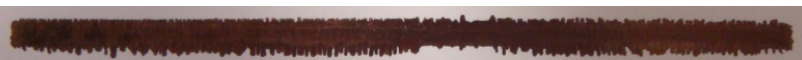

(b)

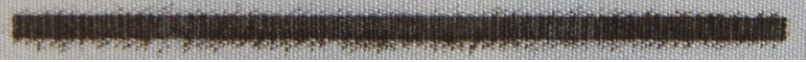

(c)

Figure 5: Examples of inkjet printed copper layers on (a) PET, (b) paper and (c) fabric.

Fabric - The best conductivity on fabric is $1.7 \times 10^{6} \mathrm{~S} / \mathrm{m}$ obtained at $30 \mathrm{~A}$ with a $3 \mathrm{~mm} / \mathrm{s}$ curing speed.

The conductivity of the tracks using obtained using CP-004 is shown in Figure 4.

Kapton - The best conductivity on Kapton is $2.45 \times 10^{6} \mathrm{~S} / \mathrm{m}$ obtained at $33 \mathrm{~A}$ with $10 \mathrm{~mm} / \mathrm{s}$ curing speed. Printed CP-004 copper layer adhered to Kapton without any cracking/flaking.

Paper - The best conductivity on paper is $2.0 \times 10^{6} \mathrm{~S} / \mathrm{m}$ achieved at either 33 A with $10 \mathrm{~mm} / \mathrm{s}$ or 46 A with $20 \mathrm{~mm} / \mathrm{s}$. No cracks were observed.

Fabric - The best conductivity on fabric is $2.46 \times 10^{6} \mathrm{~S} / \mathrm{m}$ obtained at 33 A with $5 \mathrm{~mm} / \mathrm{s}$.

It is found that the conductivity of CP-004 on Kapton, paper and fabric is higher than that achieved using CP-003. No cracks were observed on any $\mathrm{CP}-004$ tracks. Compared with the results reported in [11] which uses laser to sinter screen printed $\mathrm{Cu}$ paste on Kapton, the conductivity achieved on the same substrate in this work is 35 times higher than the previously reported results of $7.09 \times 10^{4} \mathrm{~S} / \mathrm{m}$.

\section{B. Inkjet printed copper tracks}

After printing on all the substrates, only the tracks printed on Kapton adhered to the substrate. Figure 5 shows the

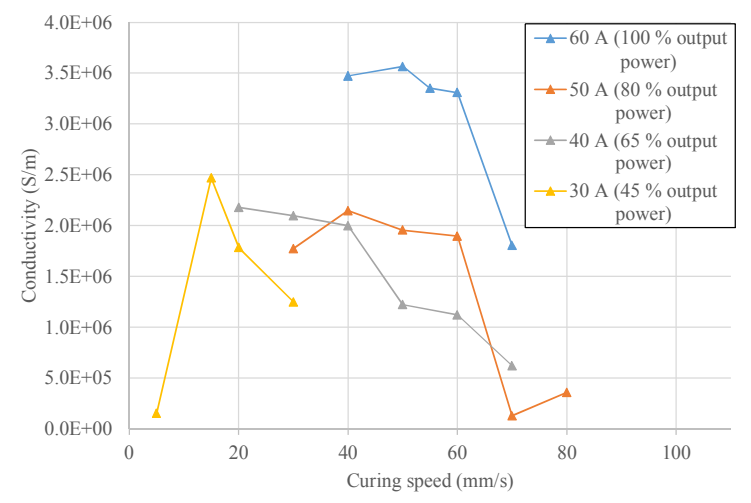

Figure 6: Conductivity vs curing speed on Kapton. 


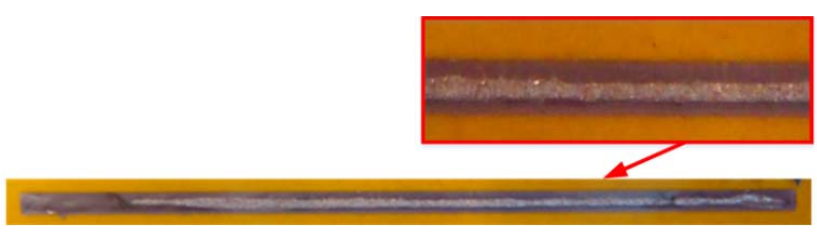

Figure 7: Example of inkjet printed copper on Kapton.

examples of the copper tracks on the other three substrates.

Since the inkjet printable ink has a low viscosity $(0.01$ $\sim 0.02$ Pa.s), the printed ink is absorbed more into both paper and fabric shown in Figure 5 (b) and (c). The laser radiation will be most intense at the surface of each substrate and therefore if the ink is strongly absorbed, the level of sintering will be reduced. Flaking of the layer on PET could be due to less binder in the ink compared to screen printing, thus reducing the surface adhesion. The measured conductivity of the tracks on Kapton is shown in Figure 6.

The best conductivity of inkjet printed copper on Kapton is $3.6 \times 10^{6} \mathrm{~S} / \mathrm{m}$ achieved at $60 \mathrm{~A}$ with $50 \mathrm{~mm} / \mathrm{s}$ curing speed. Figure 7 shows the cured copper track on Kapton.

Compared to the results reported in [12] which uses the same ink CP-002 ink-jet printed on Kapton, the achieved conductivity (i.e. $3.6 \times 10^{6} \mathrm{~S} / \mathrm{m}$ ) is eight times lower than that of $2.98 \times 10^{6} \mathrm{~S} / \mathrm{m}$ but it is believed that the discrepancy might be the difference in the laser source as well as the curing speed.

\section{CONCLUSIONS}

Both screen/inkjet printable copper NP inks have been printed onto Kapton, PET, paper and fabric and the conductivity measured after laser curing. Of the screenprintable ink, paper produces the lowest conductivity because of absorption and the polyester/cotton the highest conductivity. It is found that the fabric used is less absorbent compared with the paper and the screen-printed ink remains more on the surface rather than being absorbed. Due to the reduced absorption, more of the copper NPs would be subjected to the full laser intensity as they are at a higher concentration on the substrate surface. Of the inkjet printable ink, only the tracks on Kapton can be cured and adhere to the substrate, whereas the tracks on PET, paper and fabric cannot be cured due to either poor adhesion or excessive absorption. A lower concentration of the inkjet ink on the surface results in lower conductivity because there are less copper NPs at the surface of the substrate where the laser radiation is most intense. Therefore, there is reduced sintering of the NPs hence a less continuous conductor.

\section{FUTURE WORK}

Future work will achieve further characterisation of the printed sample in terms of bending, scratching and aging.

\section{ACKNOWLEDGEMENT}

This research was funded in part by the European 'Organic Large Area Electronics (OLAE+)' program and the Technology Strategy Board (Project No.: 18257-114240) U.K.
The authors would like to thank Intrinsiq Material Ltd for supplying the printable copper pastes.

\section{REFERENCES}

[1] [1]. London Metal Exchange. [Online]. Available: http://www.lme.com/metals/.

[2] [2]. DuPont. [Online]. Available: http://www2.dupont.com/MCM/en_US/products/cb_series/copper_cond uctive_inks.html.

[3] [3]. Novacentrix. [Online]. Available: http://www.novacentrix.com/.

[4] [4]. I. M. Ltd. [Online]. Available: http://intrinsiqmaterials.com/.

[5] [5]. J. S. Kang, H. S. Kim, J. Ryu, H. T. Hahn, S. Jang and J. W. Joung, "Inkjet printed electronics using copper nanoparticle ink," Journal of Material Science: Material Electronics, vol. 21, pp. 1213$1220,2010$.

[6] [6]. B. Xu, F. Arias and G. M. Whitesides, "Making honeycomb microcomposites by soft lithography," Advanced materials, vol. 11, pp. 492-495, 1999.

[7] [7]. Y. Kim, B. Lee, S. Yang, I. Byun, I. Jeong and S. M. Cho, "Use of copper ink for fabricating conductive electrodes and RFID antenna tags by screen printing," Current Applied Physics, vol. 12, pp. 473-478, 2012.

[8] [8]. R. Dorey, Ceramic thick film for MEMS and microdevices, Elsevier Inc..

[9] [9]. S. Hoath, G. Martin and I. Hutchings, "Effects of fluid viscosity on drop-on-demand ink-jet break-off," in NIP26 and digital fabrication, 2010.

[10] [10]. E. Fearon, T. Sato, D. Wellburn, K. G. Watkins and G. Dearden, "Thermal effects of substrate materials used in the laser curing of particular silver inks," in 5th Laser Assisted Net Shape Engineering, 2007.

[11] [11]. M. Joo, B. Lee, S. Jeong and M. Lee, "Comparative studies on thermal and laser sintering for highly conductive $\mathrm{Cu}$ films printable on plastic substrate," Thin Solid Films, vol. 520, pp. 2878-2883, 2012.

[12] [12]. M. Zenou, O. Ermak, A. Saar and Z. Kotler, "Laser sintering of copper nanoparticles," Journal of Physics D: Applied Physics, vol. 47, p. $025501,2014$. 